\title{
The Steglich Esterification
}

Organo- and

Biocatalysis

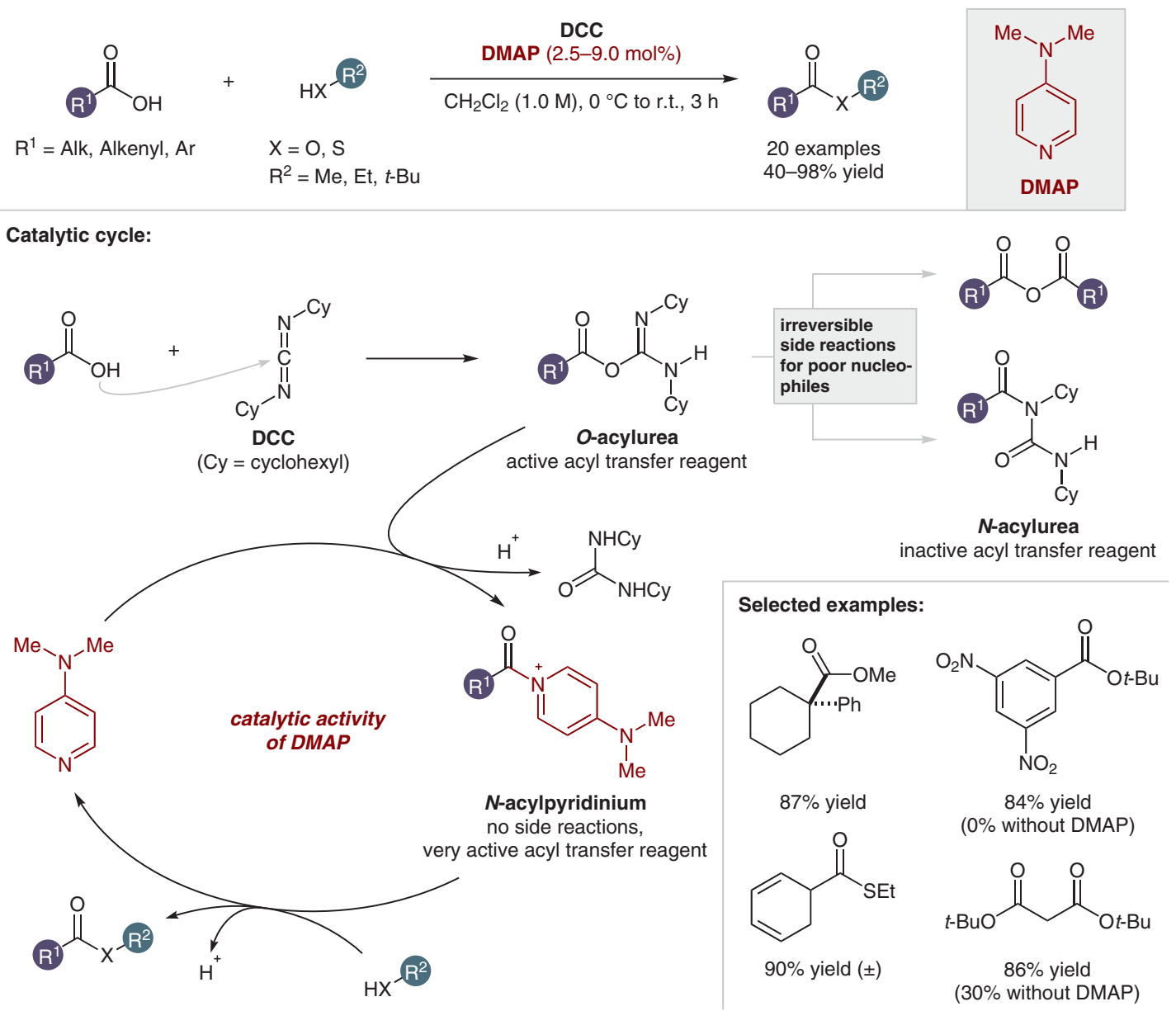

\section{Key words}

carboxylic acid activation

dimethylaminopyridines

dicyclohexylcarbodiimides

esterification

Significance: In 1978, Neises and Steglich showed that a catalytic amount of DMAP can drastically enhance reactivity in carbodiimide-activated esterification reactions. This method not only improved the yields of the products while shortening the reaction times, but also permitted the construction of, at that date, inaccessible molecules. In later years, this protocol has become one of the most frequently used esterification strategies, with a tremendous impact on both academia and industry.
Comment: In carbodiimide-mediated carboxylic acid activation reactions, the corresponding $\mathrm{O}$-acylurea is the most relevant intermediate. When poor nucleophiles are used, side reactions prevail and lead to diminished yields or complete lack of productive reactivity. DMAP (previously introduced by Litvinenko and Kirichenko, see: Dok. Akad. Nauk SSSR, Ser. Khim. 1967, 176, 97) directly accepts the acyl group from the $O$-acylurea, thereby preventing possible side reactions and effectively transferring it to the respective nucleophile. 